PROCEEDINGS OF THE

AMERICAN MATHEMATICAL SOCIETY

Volume 128, Number 12, Pages 3639-3646

S 0002-9939(00)05516-7

Article electronically published on June 7, 2000

\title{
ONE-STEP EXTENSION OF THE BERGMAN SHIFT
}

\author{
YONG BIN CHOI, JIN KYU HAN, AND WOO YOUNG LEE
}

(Communicated by David R. Larson)

\begin{abstract}
In this paper we answer a question of Curto and Fialkow: there exists a quadratically hyponormal weighted shift which is not positively quadratically hyponormal.
\end{abstract}

Let $\mathcal{H}$ be a complex Hilbert space and let $\mathcal{L}(\mathcal{H})$ be the algebra of bounded linear operators on $\mathcal{H}$. An operator $T \in \mathcal{L}(\mathcal{H})$ is said to be normal if $T^{*} T=T T^{*}$, hyponormal if $T^{*} T \geq T T^{*}$, and subnormal if $T=\left.N\right|_{\mathcal{H}}$, where $N$ is normal on some Hilbert space $\mathcal{K} \supseteq \mathcal{H}$. If $T$ is subnormal, then $T$ is also hyponormal. Recall that given a bounded sequence of positive numbers $\alpha: \alpha_{0}, \alpha_{1}, \cdots$ (called weights), the (unilateral) weighted shift $W_{\alpha}$ associated with $\alpha$ is the operator on $\ell^{2}\left(\mathbb{Z}_{+}\right)$defined by $W_{\alpha} e_{n}:=\alpha_{n} e_{n+1}$ for all $n \geq 0$, where $\left\{e_{n}\right\}_{n=0}^{\infty}$ is the canonical orthonormal basis for $\ell^{2}$. It is straightforward to check that $W_{\alpha}$ can never be normal, and that $W_{\alpha}$ is hyponormal if and only if $\alpha_{n} \leq \alpha_{n+1}$ for all $n \geq 0$.

Recall the Bram-Halmos criterion for subnormality, which states that an operator $T \in \mathcal{L}(\mathcal{H})$ is subnormal if and only if

$$
\sum_{i, j}\left(T^{i} x_{j}, T^{j} x_{i}\right) \geq 0
$$

for all finite collections $x_{0}, x_{1}, \cdots, x_{k} \in \mathcal{H}$ ([1, 2, III.1.9]). Using the Choleski algorithm for operator matrices, it is easy to see that this is equivalent to the positivity of the matrices $\left(T^{* j} T^{i}-T^{i} T^{* j}\right)_{i, j=1}^{k}$ for $k=1,2, \cdots$. If we denote by $[A, B]:=A B-B A$ the commutator of two operators $A$ and $B$, and if we define $T$ to be $k$-hyponormal whenever the $k \times k$ operator matrix $M_{k}(T):=\left(\left[T^{* j}, T^{i}\right]\right)_{i, j=1}^{k}$ is positive, then the Bram-Halmos criterion can be rephrased as saying that $T$ is subnormal if and only if $T$ is $k$-hyponormal for every $k \geq 1$ ([7]). Recall ([3], [4]) that $T \in \mathcal{L}(\mathcal{H})$ is weakly k-hyponormal if $\sum_{i=0}^{k} s_{i} T^{i}$ is hyponormal for every complex number $s_{i}(0 \leq i \leq k)$. If $k=2$, then it is said to be quadratically hyponormal. It is known that 2-hyponormal $\Rightarrow$ quadratically hyponormal. In [3] Proposition 7], it is shown that there exists a quadratically hyponormal weighted shift which is not 2-hyponormal.

Received by the editors October 4, 1998 and, in revised form, February 24, 1999.

1991 Mathematics Subject Classification. Primary 47B20, 47B37.

Key words and phrases. Quadratically hyponormal operators, positively quadratically hyponormal operators, unilateral weighted shifts, Bergman shifts.

This work was partially supported by the KOSEF through the GARC at Seoul National University and a research grant BSRI-1998-015-D00028. 
Let $W_{\alpha}$ be a hyponormal weighted shift. We write $D(s):=\left[\left(W_{\alpha}+s W_{\alpha}^{2}\right)^{*}, W_{\alpha}+\right.$ $\left.s W_{\alpha}^{2}\right]$ for $s \in \mathbb{C}$, and we let

$$
D_{n}(s):=P_{n}\left[\left(W_{\alpha}+s W_{\alpha}^{2}\right)^{*}, W_{\alpha}+s W_{\alpha}^{2}\right] P_{n},
$$

where $P_{n}$ is the orthogonal projection onto the subspace generated by $\left\{e_{0}, \cdots, e_{n}\right\}$. Then $D_{n}(s)$ is of the form

$$
D_{n}(s)=\left(\begin{array}{cccccc}
q_{0} & \bar{r}_{0} & 0 & \ldots & 0 & 0 \\
r_{0} & q_{1} & \bar{r}_{1} & \ldots & 0 & 0 \\
0 & r_{1} & q_{2} & \ldots & 0 & 0 \\
\vdots & \vdots & \vdots & \ddots & \vdots & \vdots \\
0 & 0 & 0 & \ldots & q_{n-1} & \bar{r}_{n-1} \\
0 & 0 & 0 & \ldots & r_{n-1} & q_{n}
\end{array}\right),
$$

where

$$
\begin{aligned}
q_{n} & :=u_{n}+|s|^{2} v_{n}, \\
r_{n} & :=s \sqrt{w_{n}} \\
u_{n} & :=\alpha_{n}^{2}-\alpha_{n-1}^{2} \\
v_{n} & :=\alpha_{n}^{2} \alpha_{n+1}^{2}-\alpha_{n-1}^{2} \alpha_{n-2}^{2}, \\
w_{n} & :=\alpha_{n}^{2}\left(\alpha_{n+1}^{2}-\alpha_{n-1}^{2}\right)^{2}
\end{aligned}
$$

and, for notational convenience, $\alpha_{-2}=\alpha_{-1}=0$. Clearly, $W_{\alpha}$ is quadratically hyponormal if and only if $D_{n}(s) \geq 0$ for every $s \in \mathbb{C}$ and every $n \geq 0$. Let $d_{n}(\cdot):=\operatorname{det}\left(D_{n}(\cdot)\right)$. Then $d_{n}$ satisfies the following 2-step recursive formula:

$$
d_{0}=q_{0}, \quad d_{1}=q_{0} q_{1}-\left|r_{0}\right|^{2}, \quad d_{n+2}=q_{n+2} d_{n+1}-\left|r_{n+1}\right|^{2} d_{n}
$$

if we let $t:=|s|^{2}$, we observe that $d_{n}$ is a polynomial in $t$ of degree $n+1$, and if we write $d_{n}=\sum_{i=0}^{n+1} c(n, i) t^{i}$, then the Maclaurin coefficients $c(n, i)$ satisfy a double-indexed recursive formula; namely

$$
\begin{gathered}
c(n+2, i)=u_{n+2} c(n+1, i)+v_{n+2} c(n+1, i-1)-w_{n+1} c(n, i-1), \\
c(n, 0)=u_{0} \cdots u_{n}, \quad c(n, n+1)=v_{0} \cdots v_{n}, \quad c(1,1)=u_{1} v_{0}+v_{1} u_{0}-w_{0}
\end{gathered}
$$

$(n \geq 0, i \geq 1)$.

We begin with:

Definition 1 (4], [5], [6]). Let $\alpha: \alpha_{0}, \alpha_{1}, \cdots$ be a weight sequence, let $W_{\alpha}$ be the corresponding weighted shift, and let $c(n, i)$ be the Maclaurin coefficients of the polynomial $d_{n}$. We say that $W_{\alpha}$ is positively quadratically hyponormal if $c(n, i) \geq 0$ for all $n, i \geq 0$ with $0 \leq i \leq n+1$, and $c(n, n+1)>0$ for all $n \geq 0$.

Clearly, positively quadratically hyponormal $\Longrightarrow$ quadratically hyponormal. In 1994, Curto and Fialkow ([4. Problem 4.7]) asked if the converse is true: if $W_{\alpha}$ is a quadratically hyponormal weighted shift, does it follow that $W_{\alpha}$ is positively quadratically hyponormal? In this paper we answer it negatively.

If the weight sequence $\alpha=\left\{\alpha_{n}\right\}_{n=0}^{\infty}$ is given by

$$
\alpha_{n}=\sqrt{\frac{n+1}{n+2}} \quad(n \geq 0),
$$

then the corresponding weighted shift is called the Bergman shift. It is well known that the Bergman shift is subnormal. 
The following is an one-step extension of the Bergman shift.

Theorem 2. For $x>0$, let $T_{x}$ be the weighted shift whose weight sequence is given by

$$
\alpha_{0}=\sqrt{x}, \quad \alpha_{n}=\sqrt{\frac{n}{n+1}}(n \geq 1) .
$$

Then we have:

(a) $T_{x}$ is positively quadratically hyponormal $\Longleftrightarrow 0<x \leq \frac{22}{47}$.

(b) $0<x \leq \frac{71}{151} \Longrightarrow T_{x}$ is quadratically hyponormal.

(c) $T_{x}$ is not quadratically hyponormal for $x=\frac{1}{2}$.

(d) $T_{x}$ is 2-hyponormal $\Longleftrightarrow 0<x \leq \frac{1}{3}$.

(e) $T_{x}$ is never subnormal for any $x>0$.

Proof. (a) We use an idea of Curto (3. Proposition 7]). Suppose $T_{x}$ is hyponormal and hence $0<x \leq \frac{1}{2}$. Write $d_{n}(t)=\sum_{i=0}^{n+1} c(n, i) t^{i}$. From (0.2) we can check directly that

$$
\begin{gathered}
\left\{\begin{array}{l}
c(0,0)=x, \\
c(0,1)=\frac{1}{2} x,
\end{array}\right. \\
\left\{\begin{array}{l}
c(1,0)=x\left(\frac{1}{2}-x\right), \\
c(1,1)=x\left(\frac{1}{3}-\frac{1}{2} x\right), \\
c(1,2)=\frac{1}{6} x,
\end{array}\right. \\
\begin{array}{l}
c(2,0)=\frac{x}{6}\left(\frac{1}{2}-x\right), \\
c(2,1)=\frac{x}{6}\left(\frac{1}{2}-x\right), \\
c(2,2)=\frac{x}{12}(1-x), \\
c(2,3)=\frac{x}{12}(1-x),
\end{array} \\
\begin{array}{l}
c(3,0)=\frac{x}{72}\left(\frac{1}{2}-x\right), \\
c(3,1)=\frac{x}{60}\left(\frac{1}{2}-x\right), \\
c(3,2)=\frac{11}{360} x\left(\frac{1}{2}-x\right), \\
c(3,3)=\frac{x}{720}(16-21 x), \\
c(3,4)=\frac{x}{45}(1-x),
\end{array}
\end{gathered}
$$

and

$$
\left\{\begin{array}{l}
c(4,0)=\frac{x}{1440}\left(\frac{1}{2}-x\right) \\
c(4,1)=\frac{x}{1080}\left(\frac{1}{2}-x\right) \\
c(4,2)=\frac{x}{480}\left(\frac{1}{2}-x\right) \\
c(4,3)=\frac{x}{8640}(22-47 x) \\
c(4,4)=x\left(\frac{1}{270}-\frac{7}{1440} x\right) \\
c(4,5)=\frac{x}{270}(1-x)
\end{array}\right.
$$

Observe

$$
c(n, i) \geq 0 \quad \text { for all } 0 \leq n, i \leq 3 \text { with } 0 \leq i \leq n+1
$$

Note that

$$
c(n, 0)=u_{0} \cdots u_{n} \geq 0 \text { for all } n \geq 0
$$

and

$$
c(n, 1)=u_{0} \cdots u_{n-1} \alpha_{n}^{2}\left(\alpha_{n+1}^{2}-\alpha_{n-1}^{2}\right) \geq 0 \quad(n \geq 2) .
$$


Claim I. $c(n, 2) \geq 0$ for all $n \geq 1$.

Proof of Claim I. Since for $n \geq 2$,

$$
\begin{aligned}
& v_{n+2} c(n+1,1)-w_{n+1} c(n, 1) \\
= & v_{n+2} u_{0} \cdots u_{n} \alpha_{n+1}^{2}\left(\alpha_{n+2}^{2}-\alpha_{n}^{2}\right)-w_{n+1} u_{0} \cdots u_{n-1} \alpha_{n}^{2}\left(\alpha_{n+1}^{2}-\alpha_{n-1}^{2}\right) \\
= & u_{0} \cdots u_{n-1}\left(v_{n+2} u_{n} \alpha_{n+1}^{2}\left(\alpha_{n+2}^{2}-\alpha_{n}^{2}\right)-w_{n+1} \alpha_{n}^{2}\left(\alpha_{n+1}^{2}-\alpha_{n-1}^{2}\right)\right) \\
= & u_{0} \cdots u_{n-1} \cdot \frac{24}{n(n+1)^{2}(n+2)^{2}(n+3)^{2}(n+4)} \geq 0,
\end{aligned}
$$

it follows that if $c(n+1,2) \geq 0$, then for $n \geq 2$,

$$
c(n+2,2)=u_{n+2} c(n+1,2)+v_{n+2} c(n+1,1)-w_{n+1} c(n, 1) \geq 0 .
$$

But since $c(n, 2) \geq 0$ for $n=1,2,3$, the above argument gives that $c(n, 2) \geq 0$ for all $n \geq 1$. This proves Claim I.

Claim II. For $n \geq 4, i \geq 4$,

$$
c(n, i)=v_{n} c(n-1, i-1) .
$$

Proof of Claim II. First of all we prove that for $n \geq 4, i \geq 1$,

$$
c(n, i)=v_{n} c(n-1, i-1)+u_{n} \cdots u_{4} h_{i} \quad \text { with } h_{i}:=u_{3} c(2, i)-w_{2} c(1, i-1) .
$$

A simple calculation shows that $u_{n+1} v_{n}=w_{n}$ for all $n \geq 3$. Thus if $n=4$,

$$
\begin{aligned}
c(4, i) & =u_{4} c(3, i)+v_{4} c(3, i-1)-w_{3} c(2, i-1) \\
& =v_{4} c(3, i-1)+u_{4}\left(u_{3} c(2, i)+v_{3} c(2, i-1)-w_{2} c(1, i-1)\right)-w_{3} c(2, i-1) \\
& =v_{4} c(3, i-1)+u_{4}\left(u_{3} c(2, i)-w_{2} c(1, i-1)\right)+\left(u_{4} v_{3}-w_{3}\right) c(2, i-1) \\
& =v_{4} c(3, i-1)+u_{4} h_{i}
\end{aligned}
$$

and a similar calculation works for the inductive step. Now

$$
h_{1}=\frac{1}{36} x\left(x-\frac{1}{2}\right), \quad h_{2}=\frac{1}{72} x\left(x-\frac{1}{2}\right), \quad h_{3}=-\frac{1}{144} x^{2}, \quad \text { and } \quad h_{i}=0 \text { for } i \geq 4,
$$

which together with (2.5) proves Claim II.

Claim III. If $c(n, 3) \geq 0$, then $c(n+1,3) \geq 0$ for $n \geq 4$.

Proof of Claim III. Since for $n \geq 4$,

$$
\begin{aligned}
& v_{n+1} c(n, 2)-w_{n} c(n-1,2) \\
= & v_{n+1}\left(u_{n} c(n-1,2)+v_{n} c(n-1,1)-w_{n-1} c(n-2,1)\right)-w_{n} c(n-1,2) \\
= & \left(v_{n+1} u_{n}-w_{n}\right) c(n-1,2)+v_{n+1}\left(v_{n} c(n-1,1)-w_{n-1} c(n-2,1)\right) \\
= & c(n-1,2) \cdot \frac{4}{n(n+1)^{2}(n+2)^{2}(n+3)}+v_{n+1} g_{n} \geq 0,
\end{aligned}
$$

where $g_{n}:=v_{n} c(n-1,1)-w_{n-1} c(n-2,1) \geq 0$ by $(2.4)$. Therefore if $c(n, 3) \geq 0$, 
then

$$
c(n+1,3)=u_{n+1} c(n, 3)+v_{n+1} c(n, 2)-w_{n} c(n-1,2) \geq 0,
$$

which proves Claim III.

It now follows from $(2.1),(2.2),(2.3)$, Claim I and Claim II that $c(n, i) \geq 0$ for all $n, i \geq 0$ with $0 \leq i \leq n+1$ if and only if $c(n, 3) \geq 0$ for all $n \geq 4$. Therefore by Claim III,

$$
c(n, i) \geq 0 \text { for all } n, i \geq 0 \Longleftrightarrow c(4,3) \geq 0 \Longleftrightarrow x \leq \frac{22}{47} .
$$

This proves statement (a).

(b) In view of (a), it suffices to show that if $\frac{22}{47}<x \leq \frac{71}{151}$, then $T_{x}$ is quadratically hyponormal. Thus suppose $\frac{22}{47}<x \leq \frac{71}{151}$. Then we have:

(i) $c(n, i) \geq 0$ for all $0 \leq n, i \leq 4$ with $0 \leq i \leq n+1$ except for $c(4,3)$;

(ii) (2.2), (2.3), Claim I, Claim II, and Claim III all in the proof of (a) hold.

Observe that

$$
\begin{aligned}
c(5,3) & =u_{5} c(4,3)+v_{5} c(4,2)-w_{4} c(3,2) \\
& =\frac{1}{30} x\left(\frac{11}{4320}-\frac{47}{8640} x\right)+\frac{1}{4200} x\left(\frac{1}{2}-x\right)-\frac{11}{64800} x\left(\frac{1}{2}-x\right) \\
& =\frac{1}{604800} x(72-151 x) .
\end{aligned}
$$

Thus $c(5,3) \geq 0$ since $x \leq \frac{71}{151}$. Therefore by Claim III,

$$
c(n, 3) \geq 0 \quad \text { for } n \geq 5 \text { and } 0<x \leq \frac{71}{151} .
$$

Thus by Claim II, we have

$$
c(n, i) \geq 0 \text { for all } n, i \geq 0 \text { except for } c(n, n-1)(n \geq 4) .
$$

Note that $c(4,3)<0$. Again by Claim II,

$$
c(n, n-1)<0 \quad \text { for all } n \geq 4 .
$$

Note that $d_{n}(t) \geq 0$ for $n=0,1,2,3$. Observe by Claim II that if $n \geq 6$, then

$$
\begin{aligned}
& c(n, n-2) t^{n-2}+c(n, n-1) t^{n-1}+c(n, n) t^{n} \\
& \quad=v_{n} \cdots v_{6} t^{n-5}\left(c(5,3) t^{3}+c(5,4) t^{4}+c(5,5) t^{5}\right) .
\end{aligned}
$$

Thus if $c(5,3) t^{3}+c(5,4) t^{4}+c(5,5) t^{5} \geq 0$ for every $t \geq 0$, then $d_{n}(t) \geq 0$ for every $n \geq 6$ and every $t \geq 0$ because other Maclaurin coefficients are nonnegative. Thus it will suffice to show that if $\frac{22}{47}<x \leq \frac{71}{151}$, then

$$
c(n, n-2) t^{n-2}+c(n, n-1) t^{n-1}+c(n, n) t^{n} \geq 0 \quad \text { for } n=4,5
$$

(this also implies that $d_{n}(t) \geq 0$ for $n=4,5$ ). 
Claim IV. If $\frac{22}{47}<x \leq \frac{71}{151}$, then

$$
c(4,2) t^{2}+c(4,3) t^{3}+c(4,4) t^{4} \geq 0 \text { for every } t \geq 0 .
$$

Proof of Claim IV. There are two cases to consider.

Case $1\left(0<t \leq \frac{7}{24}\right)$. From $(0.2)$ we have

$$
u_{5} c(4,3)+v_{5} c(4,2)=c(5,3)+w_{4} c(3,2) .
$$

Since by $(2.6), c(5,3)+w_{4} c(3,2) \geq 0$ we have

$$
u_{5} c(4,3)+v_{5} c(4,2) \geq 0,
$$

so that

$$
c(4,2)+\frac{7}{24} c(4,3) \geq 0 .
$$

Since $c(4,2) \geq 0$ and $c(4,3)<0$, it follows that if $0<t \leq \frac{7}{24}$, then $c(4,2)+c(4,3) t \geq$ 0 . Since $c(4,4) \geq 0$ we have that $c(4,2) t^{2}+c(4,3) t^{3}+c(4,4) t^{4} \geq 0$.

Case $2\left(t \geq \frac{7}{24}\right)$. From (0.2) we have

$$
u_{5} c(4,4)+v_{5} c(4,3)=c(5,4)+w_{4} c(3,3) .
$$

A straightforward calculation shows that if $x \leq \frac{71}{151}$, then

$$
\begin{aligned}
c(5,4)+w_{4} c(3,3) & =\frac{11}{37800} x\left(1-\frac{47}{22} x\right)+\frac{1}{180} x\left(\frac{1}{45}-\frac{7}{240} x\right) \\
& =\frac{47}{113400} x\left(1-\frac{711}{376} x\right) \geq 0 .
\end{aligned}
$$

Thus $u_{5} c(4,4)+v_{5} c(4,3) \geq 0$, so that $c(4,3)+\frac{7}{24} c(4,4) \geq 0$, which implies that if $t \geq \frac{7}{24}$, then $c(4,3)+c(4,4) t \geq 0$. Since $c(4,2) \geq 0$ we have that $c(4,2) t^{2}+$ $c(4,3) t^{3}+c(4,4) t^{4} \geq 0$.

Claim V. If $\frac{22}{47}<x \leq \frac{71}{151}$, then

$$
c(5,3) t^{3}+c(5,4) t^{4}+c(5,5) t^{5} \geq 0 \quad \text { for every } t \geq 0 .
$$

Proof of Claim V. From (0.2) we have

$$
\left\{\begin{array}{l}
u_{6} c(5,4)+v_{6} c(5,3)=c(6,4)+w_{5} c(4,3), \\
u_{6} c(5,5)+v_{6} c(5,4)=c(6,5)+w_{5} c(4,4) .
\end{array}\right.
$$

By the same argument as in Claim IV, it suffices to show that if $x \leq \frac{71}{151}$, then

$$
u_{6} c(5,4)+v_{6} c(5,3) \geq 0 \quad \text { and } \quad u_{6} c(5,5)+v_{6} c(5,4) \geq 0 .
$$

Indeed, a straightforward calculation shows that if $x \leq \frac{71}{151}$, then

$$
\begin{aligned}
c(6,4)+w_{5} c(4,3) & =x\left(\frac{1}{100800}-\frac{151}{7257600} x\right)+\frac{2}{735} x\left(\frac{11}{4320}-\frac{47}{8640} x\right) \\
& =\frac{107}{6350400} x\left(1-\frac{1809}{856} x\right) \geq 0
\end{aligned}
$$


and

$$
\begin{aligned}
c(6,5)+w_{5} c(4,4) & =x\left(\frac{11}{453600}-\frac{47}{907200} x\right)+\frac{2}{735} x\left(\frac{1}{270}-\frac{7}{1440} x\right) \\
& =\frac{109}{3175200} x\left(1-\frac{413}{218} x\right) \geq 0,
\end{aligned}
$$

which together with (2.9) proves (2.10) and hence Claim V. Now Claim IV and Claim V prove (2.8); therefore if $0<x \leq \frac{71}{151}$, then $T_{x}$ is quadratically hyponormal.

(c) Observe that if $x=\frac{1}{2}$, then

$$
d_{4}(t)=\frac{1}{1080} t^{5}+\frac{11}{17280} t^{4}-\frac{1}{11520} t^{3}
$$

so that

$$
\lim _{t \rightarrow 0+} \frac{d_{4}(t)}{t^{3}}=-\frac{1}{11520}<0,
$$

which implies that $T_{x}$ is not quadratically hyponormal.

(d) Remember (3, Corollary 5]) that if $W_{\alpha}$ is the weighted shift with weights $\alpha=\left\{\alpha_{n}\right\}_{n=0}^{\infty}$, then $W_{\alpha}$ is 2-hyponormal if and only if

$$
\alpha_{n+1}^{2}\left(\alpha_{n+2}^{2}-\alpha_{n}^{2}\right)^{2} \leq\left(\alpha_{n+1}^{2}-\alpha_{n}^{2}\right)\left(\alpha_{n+2}^{2} \alpha_{n+3}^{2}-\alpha_{n}^{2} \alpha_{n+1}^{2}\right) \quad(n \geq 0) .
$$

Thus $T_{x}$ is 2-hyponormal if and only if

$$
\alpha_{1}^{2}\left(\alpha_{2}^{2}-x\right)^{2} \leq\left(\alpha_{1}^{2}-x\right)\left(\alpha_{2}^{2} \alpha_{3}^{2}-x \alpha_{1}^{2}\right) ;
$$

that is,

$$
\frac{1}{2}\left(\frac{2}{3}-x\right)^{2} \leq\left(\frac{1}{2}-x\right)\left(\frac{1}{2}-\frac{1}{2} x\right)
$$

or equivalently, $0<x \leq \frac{1}{3}$.

(e) Let $W_{\alpha}$ be the weighted shift with weights $\alpha=\left\{\alpha_{n}\right\}_{n=0}^{\infty}$ and let $\beta_{n}:=$ $\alpha_{0}^{2} \cdots \alpha_{n-1}^{2}$ for $n \geq 1$. Then C. Berger's characterization of subnormality for unilateral weighted shifts (cf. [2, III.8.16]) states that $W_{\alpha}$ is subnormal if and only if there exists a Borel probability measure $\mu$ supported on $\left[0,\left\|W_{\alpha}\right\|^{2}\right]$, with $\left\|W_{\alpha}\right\|^{2} \in \operatorname{supp} \mu$ such that

$$
\beta_{n}=\int_{0}^{\left\|W_{\alpha}\right\|^{2}} t^{n} d \mu(t) \quad \text { for all } n \geq 1 .
$$

By an argument of Curto [3] Proposition 8], if $W_{\alpha}$ is a weighted shift whose restriction to $\bigvee\left\{e_{1}, e_{2}, \cdots\right\}$ is subnormal, with associated measure $\mu$, then $W_{\alpha}$ is subnormal if and only if

(i) $\frac{1}{t} \in L^{1}(\mu)$;

(ii) $\alpha_{0}^{2} \leq\left(\left\|\frac{1}{t}\right\|_{L^{1}(\mu)}\right)^{-1}$.

A straightforward calculation shows that the Bergman shift has measure $d \mu=d t$. Indeed, $\beta_{n}=\frac{1}{n+1}=\int_{0}^{1} t^{n} d \mu(t)$ has a solution $d \mu=d t$. Thus $\frac{1}{t}$ is not integrable with respect to $\mu$. This implies that $T_{x}$ is never subnormal for any $x>0$.

Corollary 3. Let $T_{x}$ be as in Theorem 2. If $\frac{22}{47}<x \leq \frac{71}{151}$, then $T_{x}$ is quadratically hyponormal and not positively quadratically hyponormal. 


\section{ACKNOWLEDGEMENTS}

The authors are thankful to Professor R.E. Curto for many helpful comments and discussions.

\section{REFERENCES}

1. J. Bram, Subnormal operators, Duke Math. J. 22 (1955), 75-94. MR 16:835a

2. J.B. Conway, Subnormal Operators, Pitman Publ. Co., London, 1981. MR 83i:47030

3. R.E. Curto, Quadratically hyponormal weighted shifts, Int. Eq. Op. Th. 13 (1990), 49-66. MR 90k:47061

4. R.E. Curto, Joint hyponormality: A bridge between hyponormality and subnormality, Proc. Symposia Pure Math. 51 (1990), Part II, 69-91. MR 91k:47049

5. R.E. Curto and L.A. Fialkow, Recursively generated weighted shifts and the subnormal completion problem, II, Int. Eq. Op. Th. 18 (1994), 369-426. [MR 94m:47044]

6. R.E. Curto and I.B. Jung, Quadratically hyponormal weighted shifts with two equal weights, preprint.

7. R.E. Curto, P.S. Muhly and J. Xia, Hyponormal pairs of commuting operators, Operator Theory: Adv. Appl. 35 (1988), 1-22. MR 90m:47037

Department of Mathematics, Sungkyunkwan University, Suwon 440-746, Korea

Department of Mathematics Education, Mokwon University, Daejon 301-719, Korea

Department of Mathematics, Sungkyunkwan University, Suwon 440-746, Korea

E-mail address: wylee@yurim.skku.ac.kr 